\title{
Clinical severity of pediatric respiratory illness with enterovirus D68 compared with rhinovirus or other enterovirus genotypes
}

\author{
Dominik Mertz MD MSc, Abdulsalam Alawfi MD, Jeffrey M. Pernica MD MSc, Candy Rutherford MLT ART, \\ Kathy Luinstra BSc, Marek Smieja MD PhD
}

See also www.cmaj.ca/lookup/doi/10.1503/cmaj.151121

\section{- Abstract \\ Background: Enterovirus D68 (EV-D68) resulted in a reported increase in the number of children needing hospital or critical care admission because of respiratory insufficiency during 2014. It remains unclear, however, whether EV-D68 infections were more severe than rhinovirus or non-EV-D68 enterovirus infections.}

Methods: We evaluated consecutive children presenting to a pediatric hospital between Aug. 1 and Oct. 31, 2014, with positive nasopharyngeal swabs for rhinovirus or enterovirus that were sent automatically for EV-D68 testing. We compared characteristics and outcomes of patients with EV-D68 with those with rhinovirus or nonEV-D68 enterovirus in a matched cohort study.

Results: A total of 93/297 (31.3\%) of rhinovirus or enterovirus samples tested positive for
EV-D68, and it was possible to compare 87 matched pairs. Children with EV-D68 infection were more likely to have difficulty breathing (odds ratio [OR] 3.00, 95\% confidence interval $[\mathrm{Cl}]$ 1.47-6.14). There was no significant difference in admission to the critical care unit or death among children with EV-D68 infection compared with those with other rhinovirus or enterovirus infections (adjusted OR 1.47, $95 \% \mathrm{Cl}$ 0.61-3.52). Children with EV-D68 infection were more often admitted to hospital, but not significantly so (adjusted OR $2.29,95 \% \mathrm{Cl}$ 0.96-5.46).

Interpretation: Enterovirus D68 seems to be a more virulent pulmonary pathogen than rhinovirus or non-EV-D68 enterovirus, but we did not find a significant difference in death or need for critical care.
$\mathrm{R}$ hinoviruses and enteroviruses are closely related, small RNA viruses in the genus Enterovirus. Rhinovirus species are the leading cause of upper respiratory tract disease, whereas enterovirus species generally infect the gastrointestinal tract and may then disseminate systemically. In August of 2014, the first reports of severe respiratory illness caused by enterovirus D68 (EV-D68), described in the media as a "new" pathogen, came from the American Midwest. ${ }^{1}$ However, it should be emphasized that EV-D68 has been circulating for decades. It was first detected in 1962 in California, ${ }^{2}$ and has since been detected in several countries, including the Philippines, Japan and the Netherlands, as well as in previous clusters in the United States. ${ }^{3-5}$ However, by the end of 2014 , a total of more than 1000 cases from 49 states in the US were reported. ${ }^{6}$ Almost $40 \%$ of samples submitted to the Centers for Disease Control and Prevention (CDC) for suspected EV-D68 were positive for
EV-D68, ${ }^{6}$ and the genotype spread further north into Canada. ${ }^{7}$

Subsequent to media reports, numerous clinicians perceived an "increase" in the number of children who required hospital or intensive care admission for respiratory disease, leading to the hypothesis that EV-D68 was more pathogenic than other respiratory viral pathogens. However, this has not yet been determined. The CDC documented a total of 12 deaths under investigation in 1152 confirmed cases for an estimated mortality of $1 \%,{ }^{6}$ which does not appear to greatly exceed expected mortality associated with rhinovirus or non-EV-D68 enterovirus infection. ${ }^{8}$ Furthermore, the CDC estimates may have been artificially elevated by spectrum bias, as patients with severe illness would be more likely to have specimens forwarded to reference laboratories for EV-D68 testing.

The goal of this study was to compare the severity of illness in children with EV-D68
Competing interests: None declared.

This article has been peer reviewed.

Accepted: Sept. 9, 2015

Online: Oct. 13, 2015

Correspondence to: Dominik Mertz, mertzd@mcmaster.ca

CMAJ 2015. DOI:10.1503/ cmaj.150619 
infection with that of children with rhinovirus or non-EV-D68 enterovirus infection.

\section{Methods}

\section{Study setting}

This study was conducted at McMaster Children's Hospital (MCH) in Hamilton, Ontario, which has a catchment area population of 2.3 million residents. The hospital is an acute care and tertiary care centre with 159 beds, and about 6500 hospital admissions and 40000 emergency department visits annually. The study was approved by the Hamilton Integrated Research Ethics Board.

\section{Study design}

Children admitted at $\mathrm{MCH}$ with respiratory symptoms routinely have nasopharyngeal swabs tested for viral respiratory pathogens using a multiplex polymerase chain reaction (PCR) platform that includes a target for rhinovirus and enterovirus. Screening for respiratory virus in children seen and discharged home from the $\mathrm{MCH}$ emergency department is performed at the discretion of the treating physician. The study cohort included all children less than 18 years of age who presented to the $\mathrm{MCH}$ emergency department or who were admitted to hospital and were found to have a positive PCR test for rhinovirus or enterovirus between Aug. 1 and Oct. 31, 2014. Testing for EV-D68 was developed in mid-September 2014 and was performed retrospectively at that time and prospectively thereafter. No cases of EV-D68 were detected from Nov. 1 to 16, 2014, and routine EV-D68 testing was discontinued thereafter. Therefore, we included only patients tested up until Oct. 31. All patients with EV-D68 infection were included. For each patient with EV-D68 infection, a matched control was sought from the larger cohort of children with a nasopharyngeal swab that tested positive for rhinovirus or enterovirus but negative for EV-D68. Controls were matched $1: 1$ by sex, age $( \pm 1$ mo difference for patients aged $<6 \mathrm{mo}, \pm 6 \mathrm{mo}$ for patients aged 6-12 mo, $\pm 15 \mathrm{mo}$ for patients aged $1-3 \mathrm{yr}, \pm 2 \mathrm{yr}$ for patients aged 3-6 yr, and any age difference with the closest control chosen for patients aged $\geq 6 \mathrm{yr}$ ) and date of presentation. A suitable control could not be found for 6 patients, who were therefore excluded from the analysis.

\section{Laboratory testing}

All specimens were collected with flocked nasopharyngeal swabs into Universal Transport Medium (UTM, Copan Italia), extracted with easyMAG automated extraction (bioMérieux), and run by real-time multiplex PCR on the RotorGene 6500 (QIAGEN). Virus testing was done using 2 laboratory-developed and -validated multiplex PCR assays (C.R., unpublished data, 2013). The first multiplex PCR targets influenza, respiratory syncytial virus, rhinovirus and enterovirus, and a second targets human parainfluenza types 1-3, human metapneumovirus and adenovirus. The rhinovirus-enterovirus PCR target lies in the $5^{\prime}$ untranslated region of the virus.

In September 2014, all nasopharyngeal swabs that tested positive for rhinovirus or enterovirus were further tested using a novel laboratorydeveloped PCR assay specific for EV-D68. The assay targets a unique sequence within the VP1 gene, and showed high analytical sensitivity with titrated reference EV-D68 material obtained from the National Microbiology Laboratory in Winnipeg, Manitoba. Both the EV-D68-specific assay and the rhinovirus-enterovirus component of the multiplex assay have a limit of detection of 306 copies/mL of EV-D68 Fermon strain. Multiplex PCR testing of nasopharyngeal swabs is performed twice daily, with rhinovirus and enterovirus results available within 12-24 hours of specimen submission. Testing for EV-D68 was not available routinely until Sept. 29, 2014, after which date results were reported within 48 hours of specimen submission.

\section{Outcomes}

The primary outcome was a composite of indicators for severe disease consisting of admission to the pediatric critical care unit and/or death during the hospital stay. Deaths clearly unrelated to the viral infection (i.e., that occurred $>14$ days after the positive nasopharyngeal swab and did not appear to be clinically related) were excluded. Secondary outcomes included admission to hospital, the individual components of the composite outcome, need for invasive or noninvasive mechanical ventilation, length of stay in hospital or in the pediatric critical care unit, need for supplemental oxygen at any point, and the need for either magnesium sulfate or intravenous salbutamol therapy.

\section{Statistical analysis}

Patients with EV-D68 infection were compared with a 1:1 matched control group with patients who had a positive PCR test for rhinovirus or enterovirus but a negative test for EV-D68. The McNemar test and the paired $t$ test were used as appropriate for univariable analysis. Assuming a nonnormal distribution for length-of-stay data, the Wilcoxon signed rank test was used to compare between groups. Covariables with a difference between the 2 groups with a $p$ value of $<0.2$ in univariable analysis were included in a forward stepwise multivariable conditional logistic regression model for the primary outcome as well as for 
secondary outcomes. Symptoms at presentation were not included in the multivariable conditional regression because of collinearity with the primary outcome as well as other outcomes; for example, increased work of breathing would be strongly and directly related to the probability of being admitted to hospital, receiving intravenous magnesium sulfate or being admitted to the critical care unit. In the regression analyses, allergy was dichotomized (i.e., absence/presence of a history of any type of atopic disease or eosinophilia detected at the time of infection), as was the presence of other medical comorbidities (i.e., yes/no for any comorbidity). The analyses were performed using PASW Statistics 18 (IBM) and Stata 11.2 (StataCorp).

\section{Results}

Out of 297 samples that tested positive for rhinovirus or enterovirus between August and October

Table 1: Characteristics and symptoms of 87 patients with enterovirus D68 and 87 matched controls with rhinovirus or other strains of enterovirus

\begin{tabular}{|c|c|c|c|}
\hline \multirow[b]{2}{*}{ Characteristic } & \multicolumn{2}{|c|}{ No. $(\%) *$} & \multirow[b]{2}{*}{ OR $(95 \% \mathrm{Cl}) * \dagger$} \\
\hline & EV-D68 & Non-EV-D68 & \\
\hline Age, mean, $m o \pm S D$ & $57.9 \pm 47.3$ & $58.0 \pm 48.8$ & Matching variable \\
\hline Sex, female & $28(32.2)$ & $28(32.2)$ & Matching variable \\
\hline (History of) fever $¥$ & $51(58.6)$ & $51(58.6)$ & $1.00(0.53-1.89)$ \\
\hline Viral coinfections§ & $2(2.3)$ & $2(2.3)$ & $1.00(0.14-7.10)$ \\
\hline Invasive bacterial coinfectionๆ & $4(4.6)$ & 4 (4.6) & $1.00(0.20-4.95)$ \\
\hline \multicolumn{4}{|l|}{ Symptoms at presentation } \\
\hline Cough & $67(77.0)$ & $69(79.3)$ & $0.87(0.41-1.82)$ \\
\hline $\begin{array}{l}\text { Shortness of breath/increased work of } \\
\text { breathing/respiratory distress }\end{array}$ & $60(69.0)$ & $40(46.0)$ & $3.00(1.47-6.14)^{* *}$ \\
\hline Acute rhinitis & $23(26.4)$ & $35(40.2)$ & $0.54(0.28-1.03) \dagger \dagger$ \\
\hline Diarrhea & $2(2.3)$ & $9(10.3)$ & $0.22(0.05-1.03)+\dagger$ \\
\hline Vomiting & $15(17.2)$ & $14(16.1)$ & $1.09(0.48-2.47)$ \\
\hline $\begin{array}{l}\text { Not fully vaccinated according to provincial } \\
\text { vaccination schedule }\end{array}$ & $\begin{array}{l}n=81 \\
4 \quad(4.9)\end{array}$ & $\begin{array}{l}n=78 \\
2(2.6)\end{array}$ & $p=0.2 \dagger$ \\
\hline \multicolumn{4}{|l|}{ Allergy personal history } \\
\hline Asthma/repeated wheezing & $35(40.2)$ & $25(28.7)$ & $1.77(0.90-3.49)+\dagger$ \\
\hline Eczema & $18(20.7)$ & $11(12.6)$ & $1.70(0.78-3.71)+\dagger$ \\
\hline Hay fever & $12(13.8)$ & $10(11.5)$ & $1.22(0.51-2.95)$ \\
\hline Family history of allergy & $18(20.7)$ & $8 \quad(9.2)$ & $2.25(0.98-5.17)^{* *}$ \\
\hline Eosinophilia $>500 / \mu \mathrm{L}$ at presentation & $\begin{array}{c}n=58 \\
11(19.0)\end{array}$ & $\begin{array}{l}n=60 \\
4 \quad(6.7)\end{array}$ & $4.50(0.97-20.8)$ ** \\
\hline Other comorbidities & $24(27.6)$ & $35(40.2)$ & $0.59(0.32-1.10)+\dagger$ \\
\hline Neuromuscular disease & $6(6.9)$ & $6(6.9)$ & $1.00(0.29-3.45)$ \\
\hline Nonmalignant hematologic disorder & $3(3.4)$ & $4(4.6)$ & $0.75(0.17-3.35)$ \\
\hline Malignant disease & 4 (4.6) & $11(12.6)$ & $0.30(0.08-1.09)+\dagger$ \\
\hline Premature birth & 4 (4.6) & $8 \quad(9.2)$ & $0.50(0.15-1.66)$ \\
\hline Cardiovascular disease & $2(2.3)$ & $3(3.4)$ & $0.67(0.11-3.99)$ \\
\hline Respiratory disease (other than asthma) & $0 \quad(0)$ & $8(9.2)$ & $p=0.005 \dagger$ \\
\hline \multicolumn{4}{|c|}{ 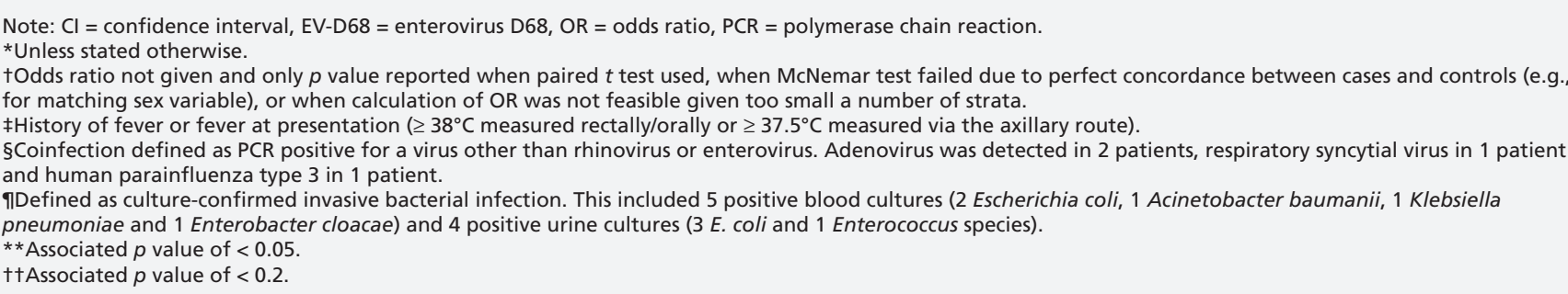 } \\
\hline
\end{tabular}


2014, 93 (31.3\%) tested positive for EV-D68. Because controls could not be found for 6 patients, there were a total of 87 matched pairs.

The groups were well matched, with a mean age of 57.9 and 58.0 months, respectively (Table 1). The number of positive results for EV-D68 and non-EV-D68 rhinovirus or enterovirus peaked by mid-September of 2014 (Figure 1). Viral and invasive bacterial coinfections were rare in both groups.

At presentation, difficulty breathing (defined as either documentation of shortness of breath or observed increased work of breathing in the patient chart) was more common in patients with EV-D68 (OR 3.00, 95\% CI 1.47-6.14) (Table 1). Children with non-EV-D68 infection appeared to more commonly exhibit acute rhinitis and diarrhea, but these differences were not significant. In addition, there were 2 cases of apnea in each group. Patients with EV-D68 tended to have a family history of allergy (OR 2.25, 95\% CI 0.98 5.17) but less significant medical comorbidities (OR 0.59, 95\% CI 0.32-1.10); the biggest differences between the groups were seen in malignant disease (OR 0.30, 95\% CI 0.08-1.09) and respiratory comorbidities other than asthma ( $0 \%$ v. $9.2 \%$, $p=0.005$ ).

Children with EV-D68 were not admitted to the pediatric critical care unit more frequently

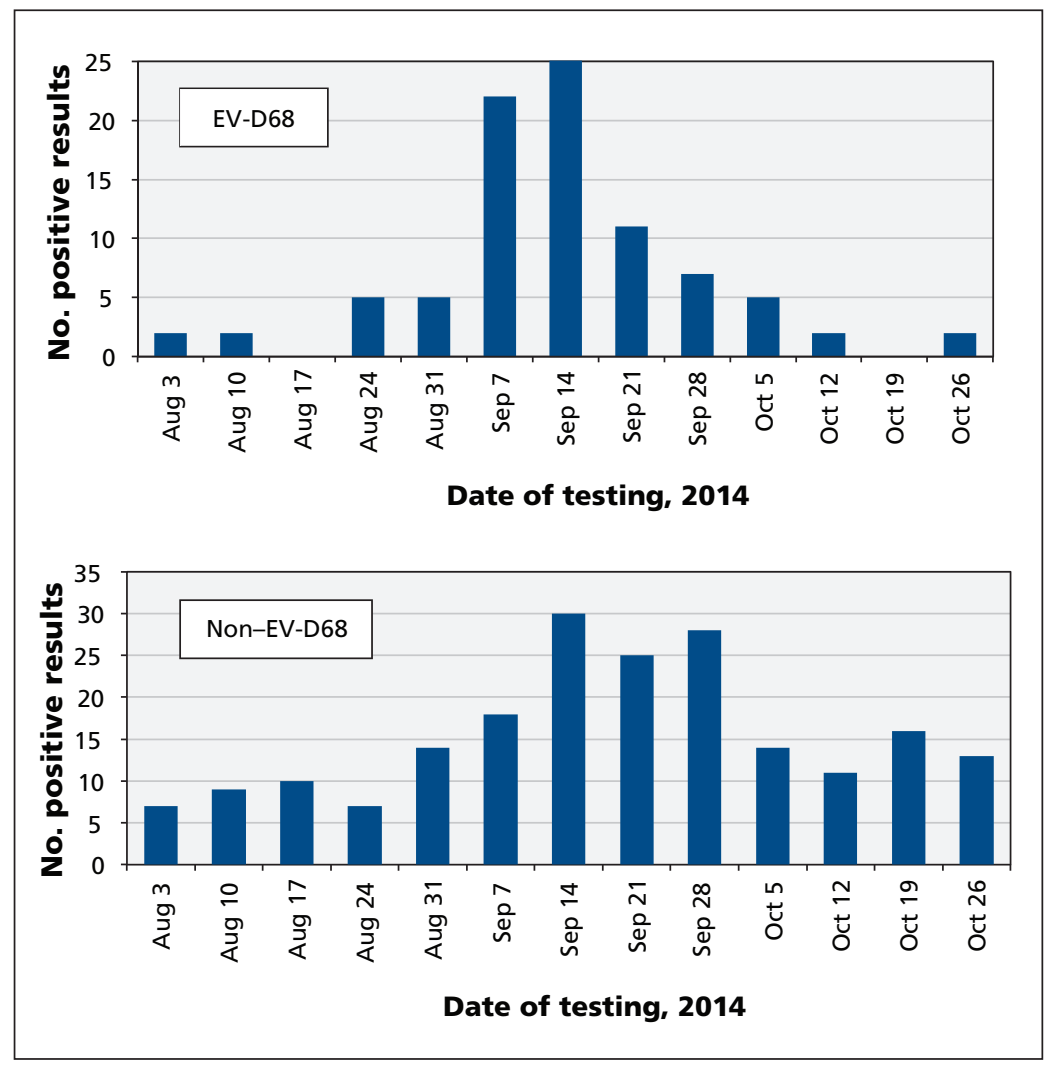

Figure 1: Positive results for enterovirus D68 (EV-D68) and non-EV-D68 rhinovirus or enterovirus, by date of nasopharyngeal swab test. and did not have higher mortality than those with non-EV-D68 infection (OR 1.78, 95\% CI 0.794.02) (Table 2). In contrast, patients with EV-D68 were more likely to be admitted to hospital (OR 2.33, 95\% CI 1.07-5.09) and were more likely to be administered magnesium sulfate (OR 3.00, 95\% CI 1.34-6.68) and/or intravenous salbutamol (OR 9.00, 95\% CI 1.1471.0). Otherwise, the outcomes were similar in the univariable analysis. The one death that occurred, in a patient without EV-D68, was unrelated to the viral infection.

Mortality and/or admission to the pediatric critical care unit were not significantly more likely to be observed in patients with EV-D68, even after adjustment for the presence of allergy and/or other medical comorbidities (Table 2). Similarly, adjustment for these covariates did not lead to any significant change in the point estimate of the association between admission to hospital and case-control status; in the multivariable model, these additional factors were not independently significant, and they did not lead to a significant improvement in the predictive power of the model. Interestingly, the association between EV-D68 and the need for treatment with magnesium sulfate was still significant after adjustment for the presence of allergy and comorbidities (adjusted OR 2.62, 95\% CI 1.06-6.47); the magnitude of association between case status and intravenous salbutamol therapy remained large but lost significance after adjustment (adjusted OR 7.15, 95\% CI 0.88-57.8).

Two cases in each group were by definition hospital-acquired, with onset of symptoms after 48 hours of hospital admission. There was no epidemiologic evidence for in-hospital transmission for the 2 EV-D68 cases (i.e., there was no known contact or exposure to a patient with EV-D68 in the hospital). Both patients had a prolonged stay at our hospital for their underlying diseases, but spent a substantial amount of time outside of the hospital and also attended large gatherings, where the transmission likely occurred.

\section{Interpretation}

We found that patients with EV-D68 infection were more likely to have a personal or family history of allergy and to present with respiratory distress than those with rhinovirus or non-EV-D68 infection. Children with EV-D68 were also more often admitted to hospital than those with rhinovirus or other strains of enterovirus, although they were no more likely to require critical care, and there was no difference in overall mortality.

The initial reports of EV-D68 infection in the fall of 2014 described 2 small cohorts of children 
admitted to hospital in Kansas City, Missouri, and Chicago, Illinois, most of whom had an atopic history and required intensive care. ${ }^{1}$ In our study, patients with EV-D68 infection were more likely to present in respiratory distress, to have a family history of allergy, to be admitted to hospital, and to receive treatment with either magnesium sulfate or intravenous salbutamol than those with rhinovirus or non-EV-D68 enterovirus infection. However, there was no significant difference between cases and controls in terms of admission to the pediatric critical care unit or death. Rates of admission to the pediatric critical care unit in our cohort were $23.0 \%$ for children with EV-D68 and $14.9 \%$ for children with other types of rhinovirus or enterovirus, which is higher than the $4 \%$ previously reported in a smaller study. ${ }^{7}$

Other studies have reported an association between documentation of respiratory EV-D68 infection and a history of asthma ${ }^{7}$ or atopy. ${ }^{9}$ In our population, those who tested positive for EV-D68 had an increased likelihood of having a personal or family history of allergic disease, although this did not reach significance. It seems reasonable to hypothesize that EV-D68 is a more virulent pulmonary pathogen to those with pre-existing atopic disease than other rhinoviruses and enteroviruses.
Furthermore, patients with non-EV-D68 infection were more likely to have nonasthmatic comorbidities of the respiratory tract and nonstatistically higher rates of malignant disease, which may point to a potential lower pathogenicity of nonEV-D68 strains.

Our results were unlikely to have been affected greatly by coinfections. Invasive bacterial infection can lead to adverse outcomes, ${ }^{10}$ and even viral coinfections may potentially affect patient outcomes ${ }^{11}$ however, both were rare events in our patient population. Despite the lack of evidence for invasive bacterial coinfection, about one-third of patients in both groups were given antibiotic treatment, in most cases because of the suspicion of bacterial pneumonia. Exclusion of patients with coinfection in a post hoc sensitivity analysis did not change our findings.

Only 2 cases of EV-D68 were by definition hospital-associated, with onset of symptoms after 24 and 40 days after admission, respectively. In both cases, no epidemiologic link to other patients with EV-D68 was found, and both patients spent substantial time outside of the hospital premises, which was considered to be the most likely source of infection. At our hospital, the only change to the infection prevention and control measures that

Table 2: Outcomes of 87 patients with enterovirus D68 and 87 matched controls with rhinovirus or other strains of enterovirus

\begin{tabular}{|c|c|c|c|c|}
\hline \multirow[b]{2}{*}{ Outcome } & \multicolumn{2}{|c|}{ No. $(\%) *$} & \multirow[b]{2}{*}{ OR $(95 \% \mathrm{Cl})$} & \multirow{2}{*}{$\begin{array}{l}\text { Adjustedt OR } \\
\qquad(95 \% \mathrm{Cl})\end{array}$} \\
\hline & EV-D68 & Non-EV-D68 & & \\
\hline $\begin{array}{l}\text { Composite of admission to the } \\
\text { critical care unit and/or death }\end{array}$ & $20(23.0)$ & $13(14.9)$ & $1.78(0.79-4.02) \ddagger$ & $1.47(0.61-3.52)$ \\
\hline LOS, median (IQR), d & $3(2-5)$ & $3(2-5)$ & NAף & NA \\
\hline Oxygen requirement & $34(39.1)$ & $25(28.7)$ & $1.52(0.83-2.82) \ddagger$ & $1.28(0.66-2.51)$ \\
\hline Need for magnesium sulfate & $28(32.2)$ & $12(13.8)$ & $3.00(1.34-6.68) \S$ & $2.62(1.06-6.47) \S$ \\
\hline Antibiotic treatment & $24(27.6)$ & $32(36.8)$ & $0.65(0.34-1.25)$ & $0.72(0.37-1.41)$ \\
\hline $\begin{array}{l}\text { Noninvasive mechanical } \\
\text { ventilation }\end{array}$ & $1(1.1)$ & 3 (3.4) & $0.33(0.04-3.20)$ & $0.27(0.03-2.79)$ \\
\hline Invasive mechanical ventilation & $1(1.1)$ & 4 (4.6) & $0.25(0.03-2.24)$ & $0.27(0.03-2.52)$ \\
\hline Death & $0(0)$ & 1 (1.1) & NA & NA \\
\hline \multicolumn{5}{|c|}{$\begin{array}{l}\text { Note: } \mathrm{Cl}=\text { confidence interval, EV-D68 = enterovirus D68, IQR = interquartile range, LOS = length of stay, NA = not applicable, OR = odds ratio. } \\
* \text { *Unless stated otherwise. } \\
\text { †Adjusted for history of allergy and/or other medical comorbidity. "History of allergy" defined by any family or personal history of atopy, including the presence } \\
\text { of blood eosinophilia. "Other medical comorbidity" defined as any comorbidity listed in Table } 1 . \\
\text { *Associated } p \text { value of }<0.2 \text {. } \\
\$ \text { Associated } p \text { value of }<0.05 \text {. } \\
\uparrow p=0.9 \text { by the paired Wilcoxon rank sum test. } \\
* * p=0.6 \text { by the paired Wilcoxon rank sum test. }\end{array}$} \\
\hline
\end{tabular}


was implemented with the surge of EV-D68 infections was that all patients with respiratory symptoms, regardless of the presence of fever, were put under contact and droplet precautions, given reports that fevers were not common with EV-D68. The lack of fever was corroborated in our study, as less than $60 \%$ of patients had a history of fever or fever at presentation. Based on our data, these measures were sufficient to prevent in-hospital transmission of EV-D68 and measures beyond this are unlikely to be beneficial.

\section{Strengths and limitations}

Our study has a number of strengths. First, it is a large cohort of patients with EV-D68 with comprehensive data available. Second, our laboratory routinely conducted PCR testing for rhinovirus and enterovirus for all patients undergoing testing for respiratory viral infection and was one of the first to develop and offer routine EV-D68 testing, which allowed us to include all consecutive patients tested for viral respiratory infection who had positive results for rhinovirus or enterovirus. Thus, our cohort is unlikely to be affected by spectrum bias and may offer a more accurate estimate of incidence and morbidity for hospital-admitted children. Third, our study is representative of the patient population presenting to a tertiary care centre during the EV-D68 outbreak. Transfers from other hospitals were rare $(n=6)$, and there was no transfer of an EV-D68 patient, thus, the patient population represents the patient mix presenting in an emergency department setting. Most important, our study included a broad spectrum of children presenting with respiratory symptoms, and we were able to conduct a direct comparison to patients with infections by other rhinovirus or enterovirus strains.

Our study also has limitations. The decision to order a nasopharyngeal swab for respiratory viruses for outpatients was at the discretion of the treating physician. However, in patients with respiratory symptoms who needed admission, a nasopharyngeal swab was considered standard of care. This may have resulted in an overestimation of the admission rates as more benign cases may not have been tested for respiratory viruses. On the other hand, awareness of EV-D68 and the association with atopy may have resulted in an increase in sampling and a potential relative oversampling in patients with known atopy. However, this was likely to have affected both groups equally and thus the bias in our results was likely to have been minimal. Furthermore, our findings may not be generalizable to less severely ill patients (e.g., patients seen by family physicians). Also, owing to small numbers of events, extensive multivariable analyses that would have included all potentially relevant covariates were impossible.

\section{Conclusion}

We found that patients with EV-D68 infection were more likely to present with respiratory distress, more likely to be admitted to hospital, and more frequently given magnesium sulfate or intravenous salbutamol than patients with rhinovirus or non-EV-D68 infection. However, they were not significantly more likely to require admission to the pediatric critical care unit or die. No EV-D68 transmissions occurred in our hospital setting.

\section{References}

1. Midgley CM, Jackson MA, Selvarangan R, et al. Severe respiratory illness associated with enterovirus D68: Missouri and Illinois, 2014. MMWR Morb Mortal Wkly Rep 2014;63:798-9.

2. Schieble JH, Fox VL, Lennette EH. A probable new human picornavirus associated with respiratory diseases. Am J Epidemiol 1967;85:297-310.

3. Centers for Disease Control and Prevention (CDC). Clusters of acute respiratory illness associated with human enterovirus 68 - Asia, Europe, and United States, 2008-2010. MMWR Morb Mortal Wkly Rep 2011;60:1301-4.

4. Imamura T, Fuji N, Suzuki A, et al. Enterovirus 68 among children with severe acute respiratory infection, the Philippines. Emerg Infect Dis 2011;17:1430-5.

5. Wang Z, Malanoski AP, Lin B, et al. Broad spectrum respiratory pathogen analysis of throat swabs from military recruits reveals interference between rhinoviruses and adenoviruses. Microb Ecol 2010;59:623-34.

6. Enterovirus D68 in the United States, 2014. Atlanta: Centers for Disease Control and Prevention; 2014. Available: www.cdc. gov/non-polio-enterovirus/outbreaks/EV-D68-outbreaks.html (accessed 2015 Jan. 6)

7. Drews SJ, Simmonds K, Usman HR, et al. Characterization of enterovirus activity, including that of enterovirus D68, in pediatric patients in Alberta, Canada, in 2014. J Clin Microbiol 2015; 53:1042-5.

8. Asner SA, Petrich A, Hamid JS, et al. Clinical severity of rhinovirus/enterovirus compared to other respiratory viruses in children. Influenza Other Respir Viruses 2014;8:436-42.

9. Bragstad K, Jakobsen K, Rojahn AE, et al. High frequency of enterovirus D68 in children hospitalised with respiratory illness in Norway, autumn 2014. Influenza Other Respir Viruses 2015; 9:59-63.

10. McCullers JA. Insights into the interaction between influenza virus and pneumococcus. Clin Microbiol Rev 2006;19:571-82.

11. Asner SA, Science ME, Tran D, et al. Clinical disease severity of respiratory viral co-infection versus single viral infection: a systematic review and meta-analysis. PLOS ONE 2014;9:e99392.

Affiliations: Departments of Medicine (Mertz, Alawfi, Smieja), Clinical Epidemiology and Biostatistics (Mertz, Smieja), Pathology and Molecular Medicine (Mertz, Smieja); Michael G. DeGroote Institute for Infectious Diseases Research (Mertz, Smieja); Department of Pediatrics (Pernica, Smieja), McMaster University; Hamilton Regional Laboratory Medicine Program (Rutherford, Smieja); Department of Laboratory Medicine (Luinstra, Smieja), St. Joseph's Healthcare, Hamilton, Ont.

Contributors: Dominik Mertz, Abdulsalam Alawfi, Jeffrey Pernica and Marek Smieja contributed to the conception and design of the study. Abdulsalam Alawfi, Candy Rutherford and Kathy Luinstra contributed to the acquisition of data. Dominik Mertz and Jeffrey Pernica contributed to the analysis and interpretation of the data, and Marek Smieja contributed to the interpretation of the data. Dominik Mertz drafted the article, which all of the authors revised. All of the authors gave final approval of the version to be published and agreed to act as guarantors of the work.

Funding: Dominik Mertz is a recipient of a Research Early Career Award from Hamilton Health Sciences Foundation (Jack Hirsh Fellowship). This study was not funded.

Acknowledgement: An abstract of this study was presented at IDWeek, San Diego, California, on Oct. 8, 2015. 